\title{
Kommentatoren/innen des Gesamtwerkes
}

Dr. iur. Marianne Andrae

em. Professorin an der Universität Potsdam

Dr. iur. Kathrin Binder

Rechtsanwältin in Wien und niedergelassene europäische Rechtsanwältin in Vaduz

Dr. iur. Markus Fehrenbach

Professor an der Ruhr-Universität Bochum

Dr. iur. Robert Freitag, Maître en droit

(Bordeaux)

Professor an der Friedrich-Alexander Universität Erlangen-Nürnberg

Dr. iur. Urs Peter Gruber

Professor an der Johannes Gutenberg Universität Mainz

Dr. iur. Bettina Heiderhoff

Professorin an der Westfälischen Wilhelms-

Universität Münster

Dr. iur. Jan von Hein

Professor an der Universität Freiburg

Dr. iur. Tobias Helms

Professor an der Universität Marburg

Christian Hertel, LL.M. (George Washington

Universität, Washington D.C.)

Notar, Weilheim i. OB

Dr. iur. Christoph Alexander Kern, LL.M. (Harvard)

Professor an der Universität Heidelberg

Dr. iur. Kathrin Kroll-Ludwigs

Professorin an der Fachhochschule Aachen

Dr. iur. Stefan Leible

Professor, Präsident der Universität Bayreuth

Dr. iur. Martin Löhnig

Professor an der Universität Regensburg

Dr. iur. Sven Loose

Rechtsanwalt bei BSKP, Dresden

Lehrbeauftragter an der Universität Leipzig

Dr. iur. Katharina Lugani

Professorin an der Heinrich-Heine-Universität

Düsseldorf

Dr. iur. Robert Magnus

Professor an der Universität Bayreuth
Dr. iur. Peter Mankowski

Professor an der Universität Hamburg

Dr. iur. Gerald Mäsch

Professor an der Westfälischen Wilhelms-

Universität Münster

Richter am Oberlandesgericht Hamm a.D.

Dr. iur. Steffen Pabst, LL.M. (Stockholm)

Konzernjurist bei der LVV Leipziger Versorgungs- und Verkehrsgesellschaft $\mathrm{mbH}$, Leipzig

Dr. iur. Peter Georg Picht, LL.M. (Yale)

Professor an der Universität Zürich

Associated Research Fellow, Max-Planck-Institut für Innovation und Wettbewerb

Dr. iur. Dr. iur. h.c. Thomas Rauscher

Professor an der Universität Leipzig

Professor h.c. an der ELTE Universität Budapest

Dipl. Math.

Dr. iur. Johannes Scheller, geb. Cziupka

Notarassessor in Hamburg

Dr. iur. Ansgar Staudinger

Professor an der Universität Bielefeld

Dr. iur. Karsten Thorn, LL.M. (Georgetown)

Professor an der Bucerius Law School Hamburg

Dr. iur. István Varga

Professor an der ELTE Universität Budapest

Rechtsanwalt bei PROVARIS, Budapest

Dipl. Phil.

Dr. iur. Matthias Weller, Mag. rer. publ.

Direktor des Instituts für deutsches und internationales Zivilverfahrensrecht an der Rheinischen Friedrich-Wilhelms-Universität Bonn

Dr. iur. Domenik Henning Wendt, LL.M.

Professor an der Frankfurt University of Applied Sciences

Dr. iur. Denise Wiedemann, LL.M. (Lissabon)

Wiss. Referentin und Leiterin des Kompetenzzentrums Lateinamerika am Max-Planck-Institut für ausländisches und internationales Privatrecht, Hamburg

Dipl. Rpfl. 